\title{
Psychotropic drug utilization and functioning in a boarding house in
} Greece

\author{
Dimitrios Petsas*1, Dimitrios Kontis², Konstantinos Parashakis², \\ Vasiliki Lilli ${ }^{1}$, Hristos Garnetas ${ }^{2}$ and Konstantinos Kontis ${ }^{2}$
}

\author{
Address: ${ }^{1}$ Promitheas - Galini A' Residential Unit, Athens, Greece and ${ }^{24}$ th Psychiatric Emergency Department - Psychiatric Hospital of Attica \\ ("Dafni"), Greece \\ ${ }^{*}$ Corresponding author
}

\author{
from International Society on Brain and Behaviour: 3rd International Congress on Brain and Behaviour \\ Thessaloniki, Greece. 28 November - 2 December 2007 \\ Published: 17 April 2008 \\ Annals of General Psychiatry 2008, 7(Suppl I):S218 doi:I0.II86/I744-859X-7-SI-S2I 8
}

This abstract is available from: http://www.annals-general-psychiatry.com/content/7/SI/S2 I 8

(c) 2008 Petsas et al.; licensee BioMed Central Ltd.

\section{Background}

A rehabilitation program in the boarding house "Galini $\mathrm{A}^{\prime \prime}$ resulted in a significant decrease in the number of psychotropic medications and a parallel increase in the functioning of patients, during the first 18 months of their arrival at the unit. The aim of this study is to define whether these achievements were maintained.

\section{Materials and methods}

All 15 residents of the unit (mean age 52.27 years, range 37-75, suffering from psychotic disorder and/or mental retardation) were recruited in the study. Functioning was assessed with the Global Assessment of Functioning (GAF) scale and data regarding the drug treatment of each patient was collected. The assessments were made at their arrival at the unit, 18 and 32 months later.

\section{Results}

The number of the psychotropic drugs administered at the first day, 18 and 36 months later were: antipsychotics: 27 22-21, classical antipsychotics: 21-10-10, atypical antipsychotics: 6-12-11, benzodiazepines 16-3-2, mood stabilizers: 8-6-6, anticholinergics: 8-2-2, antidepressants: 2-0-0, total : 61-33-31. The mean GAF score was 36,9 - 46,6 47,8 respectively.

\section{Conclusions}

The decrease in the number of psychotropic drugs was associated with an increase in the residents' global func- tioning. Both findings were observed 18 months since their arrival at the unit and maintained after 36 months. 